\title{
Preparation and characterization of interpenetrating networks based on polyacrylates and poly(lactic acid)
}

\author{
H. Kaczmarek ${ }^{*}$, I. Vuković-Kwiatkowska \\ Department of Chemistry and Photochemistry of Polymers, Faculty of Chemistry, Nicolaus Copernicus University, Torun, \\ Poland
}

Received 27 April 2011; accepted in revised form 8 August 2011

\begin{abstract}
Three different, multifunctional acrylic monomers were photopolymerized in a matrix of poly(lactic acid), PLA, using 2-hydroxy-2-methyl-1-phenyl-propan-1-one as a photoinitiator. The kinetics of the photopolymerization of monomers in PLA, studied with Fourier Transform Infrared Spectroscopy, has been compared to analogous processes of pure monomers under the same conditions (room temperature, air atmosphere). Additionally, poly(ethylene glycol) was added to acrylate/PLA blends as plasticizer.The highly crosslinked networks obtained were characterized by FTIR and optical microscopy. The amount of insoluble gel has been estimated gravimetrically. It was found that the studied systems are characterized by very high polymerization rate, moreover, efficient grafting of polyacrylates on PLA takes place. The observed morphology indicates the heterogeneity of formed networks. The glass transition temperature of PLA in studied blends has been determined by differential scanning calorimetry.
\end{abstract}

Keywords: polymer blends and alloys, interpenetrating polymer networks, poly(lactic acid), polyacrylates

\section{Introduction}

Synthetic polymers such as polyethylene, polystyrene, poly (vinyl chloride), polyacrylates, polyesters and many other commercial macromolecular products are mainly obtained from fossil fuels. They are broadly applied in practice because of their numerous advantages such as durability, flexibility, resistance to water and chemical compounds. They are lightweight and easily processed, and also have good mechanical properties as well as possibility of various modifications.

However, their utilization is not a trivial task. Problems of waste management have led researchers and manufacturers to develop effective methods of manufacturing of materials offering an alternative to conventional plastics. Moreover, the rising fossil fuel prices and shrinking of its resources motivate the investigators to search for materials from renewable resources e.g. biopolymers. Such polymers exhibit useful properties of traditional plastics and can be organically recycled because of their biodegradability. This process includes the distribution and hydrolysis of polymers in wastewater, anaerobic digestion with biogas production and decomposition with the participation of oxygen, occurring directly in soil or in compost. Production and usage of biodegradable polymers is a competitive solution to high standards of environmental protection - relatively expensive methods of mechanical recycling of packaging waste from traditional plastics and the need for their transportation, collection and segregation into homogeneous polymers $[1,2]$.

An example of biopolymer commonly used worldwide is poly (lactic acid)/ polylactide (PLA), which has excellent application properties. PLA is energy

\footnotetext{
${ }^{*}$ Corresponding author, e-mail: halina@chem.uni.torun.pl (c) BME-PT
} 
saving, non-toxic, biocompatible and biodegradable; it has good thermal processability and rheological properties. However, for special applications, it should be modified by several additives (such as fillers, fibers). Numerous recent works were devoted to PLA modification [3-5].

For instance, PLA in mixture with polysaccharides: starch and cellulose fibers lowers the production cost and reduces the time of biological decomposition. Increase of rigidity and higher heat resistance of PLA can be achieved by introduction of inorganic fillers such as talc, mica, glass. Improved resistance to tensile load is associated with the addition of rubber to PLA. Nevertheless, the problem of incompatibility occurs during mixing of PLA with other polymers such as styrene based rubbers, thus, modified polymers containing functional groups or compatibilizers have to be used additionally.

In the case of PLA modified with polyacrylates, and especially with poly(methyl methacrylate), the films obtained from these blends are transparent, have higher glass transition temperature but lower crystallinity degree in comparison to pure PLA. Polycarbonates improves PLA the thermal stability and the resistance to cracking when stretched $[1,6]$.

A common feature of polymer blends is the presence of a continuous phase of one polymer in which other components are dispersed. Depending on the degree of dispersion and on the chemical properties one can obtain real solutions or colloidal systems.

Different physical properties of compositions can be obtained by polymer mixing in the molten state. Such manufacturing depends e.g. on the ingredient types and their interactions, stability of the mixture and its morphology. Research of the crystalline phases (in the case of semicrystalline polymers), observations of the blend structure and defects, heterogeneity of multicomponent polymeric films are carried out using precise optical methods.

There is still a lack of information of the possibility of PLA modification using ultraviolet radiation, contrary to other polymers [7-9].

Photo(co)polymerization is a polymer synthesis method induced by photon absorption of the substrate. The products of photopolymerization are protective polymer coatings on the surface of various materials such as wood, metal, paper, glass, ceramics $[10,11]$. Photografting, photocrosslinking or sur- face properties modification are other broadly used processes occurring upon electromagnetic radiation. The process of initiation of photochemical reactions requires the application of efficient photoinitiators [12-14].

The chemical structure of monomer used in photopolymerization has essential influence on the properties of product obtained. Depending on the monomer functionality one can obtain linear polymers (from monomers containing one double bond or cyclic group in molecule) or the form of a spatially cross-linked networks (from multifunctional monomers) [15].

The main advantages of multifunctional monomers polymerization is high speed of process that takes place at low, room temperature in air atmosphere (without the necessity of removing oxygen) and in the absence of organic solvents. Due to these features, photochemical manufacturing of materials is classified as an environmentally friendly technology.

Poly(lactic acid) as biodegradable and commercially available polymer found numerous applications in the production of food packaging. PLA is a good candidate for replacement of poly(ethylene terephthalate), PET - basic material for drink bottles, which is not susceptible to biological decomposition. However, PLA is characterized by a relatively high gas permeability compared to PET. Thus, intensive studies are devoted to improvement of its barrier properties. One of the possibilities of PLA modification is the preparation of blends with other components. The aim of our work was to obtain and characterize the interpenetrating polymer network based on poly (lactic acid) and polyacrylates capable to crosslinking.

\section{Experimental part}

\subsection{Chemicals}

The following chemicals were used:

- polymer - poly(lactic acid) PLA (2002 D, Nature-

Works, USA) with average molecular weight 200 000;

- monomers: pentaerythritol triacrylate (PETA, $M=$ $298 \mathrm{~g} / \mathrm{mol}$ ), pentaerythritol tetraacrylate (PETeA, $M=352 \mathrm{~g} / \mathrm{mol})$, dipentaerythritol pentaacrylate (DPEPA, $M=524 \mathrm{~g} / \mathrm{mol}$ ) - all from Polyscience Inc, Warrington, PA, USA; 


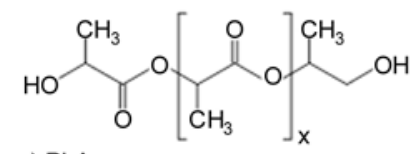

a) PLA

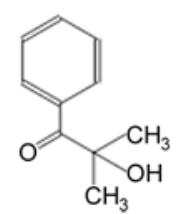

b) photoinitiator - Darocur 1173

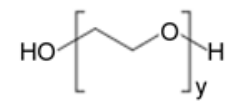

c) plasticizer - PEG 1000

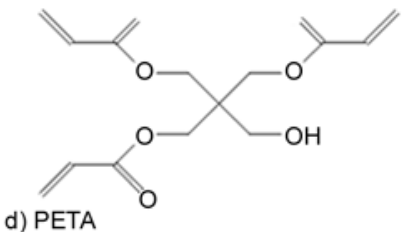

d) PETA

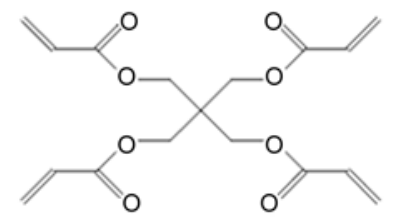

e) PETeA

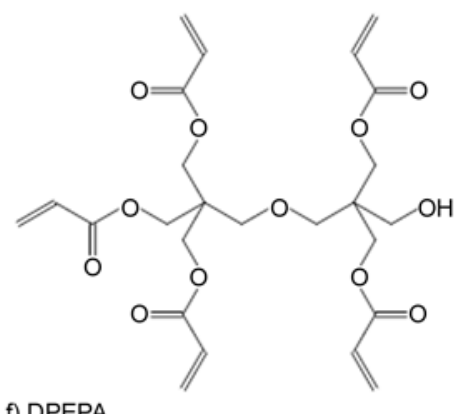

f) DPEPA

Figure 1. The chemical structure of poly(lactic acid), PLA (a); photoinitiator - Darocur 1173 (b); plasticizer - PEG (c) and monomers: PETA (d), PETeA (e) and DPEPA (f)

- photoinitiator 2-hydroxy-2-methyl-1-phenylpropan-1-one (DAROCUR 1173, Ciba, Switzerland);

- solvent - chloroform (Polish Chemical Reagents, POCH S.A., Poland),

- plasticizer - poly (ethylene glycol), PEG 1000 $\left(M_{\mathrm{v}}=1000 \mathrm{~g} / \mathrm{mol}\right.$, Polish Chemical Reagents, POCH S.A., Poland).

All materials were used as received. The chemical structures of all studied compounds are shown in Figure 1.

Number of functional groups per monomer molecule is: 3 in PETA, 4 in PETeA, 5 in DPEPA and functionality (calculated as number of $\mathrm{C}=\mathrm{C}$ bonds per $1 \mathrm{~kg}$ of monomer) is: 10.07 in PETA, 11.36 in PETeA and 9.54 in DPEPA.

\subsection{Sample preparation}

Pure PLA films were obtained from $3 \mathrm{wt} \%$ solution in chloroform. IPN films were prepared by mixing the monomers and photoinitiator with polymer solution. 1:1 weight ratio of polymer to the monomer and $5 \mathrm{wt} \%$ concentration of photoinitiator were used. After mixing, solution was poured onto leveled plates (from $\mathrm{KBr}$ for FTIR spectroscopy or from glass for microscopic observations and for determination of gel amount). Additionally, $10 \%$ wt. plasticizer was introduced for improvement of the sample flexibility. Simultaneously, the films of pure monomers with $5 \mathrm{wt} \%$ initiator were also prepared. After solvent evaporation at room temperature and careful drying (also at room temperature) in the dark, the solid blends were UV-irradiated for curing.

The samples of the same thickness $(\sim 10 \mu \mathrm{m})$ have been chosen for all experiments.

\subsection{Photopolymerization conditions}

The high pressure mercury vapor lamp, HPK $125 \mathrm{~W}$, Philips has been used for polymerization. The wavelength range and intensity of the incident light at the sample level was $248-578 \mathrm{~nm}$ and $16.65 \mathrm{~W} / \mathrm{m}^{2}$, respectively.

The following conditions were applied: time of irradiation - maximum 5 minutes, air atmosphere and room temperature $\left(20^{\circ} \mathrm{C}\right)$. The home-made device equipped with a photographic shutter has been applied for short curing times. It allows exposure to 
the sample for a split second (since 1/60 s). The polymerization kinetics were monitored by FTIR spectroscopy. The film thickness was about $0.02 \mathrm{~mm}$.

\subsection{FTIR spectroscopy}

FTIR spectra with $2 \mathrm{~cm}^{-1}$ resolution were recorded using Genesis II spectrophotometer (Mattson, USA) in range of $400-4000 \mathrm{~cm}^{-1}$. The number of scans was 32. The spectra analysis i.e. baseline correction, normalization, band intensity calculation has been done using WinFirst 3.57 software (Mattson Instruments).

The absorption band at $809 \mathrm{~cm}^{-1}$, attributed to $=\mathrm{C}-\mathrm{H}$ bending vibration, was chosen for monitoring the course of photopolymerization. The conversion degree $(X, \%)$ has been calculated by Equation (1) on the basis of the number of consumed double bonds:

Conversion degree $[\%]=\left(1-\frac{A_{\mathrm{t}}}{A_{0}}\right) \cdot 100 \%$

where $A_{\mathrm{t}} / A_{0}$ is the ratio of absorbance of the band at $809 \mathrm{~cm}^{-1}$ in the spectrum of the sample after $t$ time of reaction to the absorbance of unirradiated sample. This ratio corresponds to the amount of unreacted double bonds.

The maximum rate of polymerization was obtained from the slope of the linear part of recorded kinetic curve (where the conversion degree was plotted vs time of reaction).

\subsection{Optical microscopy}

Samples of unmodified and modified films of PLA were observed under the inverted research microscope Nikon ECLIPSE TE 2000S in the bright field technique using the Hoffman modulation contrast (HMC) allowing the enhancement of specimen contrast. The magnification was $100 \times$. The most representative images of samples were taken using a microscopic digital camera. Transfer of microscopic image on the computer screen was possible due to the specialized program of documentation and analysis of microscopic image ELEMENTS Ar NIS 2.30.

\subsection{Scanning Electron Microscopy}

Morphology of the samples was observed using LEO1430 field-emitting Scanning Electron
Microscopy at accelerating voltage of $5 \mathrm{kV}$. All specimens were coated with thin layer of gold.

\subsection{Differential Scanning Calorimetry}

DSC measurements were carried out in helium atmosphere in the temperature range of $20-250^{\circ} \mathrm{C}$ using Diamond DSC power compensation type (Perkin-Elmer). Indium was used for calibration. The following conditions were applied: flow rate $20 \mathrm{ml} / \mathrm{min}$, heating and cooling rate $-200^{\circ} \mathrm{C} / \mathrm{min}$, sample weight - ca. $5 \mathrm{mg}$. The glass transition temperature of PLA $\left(T_{\mathrm{g}}\right)$ was obtained from the inflection point on DSC curve (second run).

\subsection{Gel amount and grafting degree}

Gel content in obtained IPN films was determined gravimetrically, according to Equation (2):

$\operatorname{Gel}[\%]=\frac{m_{\mathrm{g}}}{m_{0}} \cdot 100 \%$

where $m_{\mathrm{g}}$ is weight of insoluble gel and $m_{0}$ is total weight of initial sample.

After curing and weighting, the samples were dissolved in chloroform: soluble part (sol) was extracted and separated from insoluble fraction. The extraction has been done first in chloroform at room temperature for 24 hours, then, in Soxhlet apparatus in boiling chloroform for 24 hours.

The separated gel was dried in vacuum oven to a constant weight. The amount of gel is an average of at least three values.

Taking into account that the weight ratio of PLA to acrylate was always 50:50, the amount of grafted PLA (so-called grafting degree) has been obtained from the Equation (3):

Grafting degree $[\%]=\frac{\text { gel amount }-50}{50} \cdot 100 \%$

\section{Results and discussion}

\subsection{Spectral characterization of reagents}

FTIR spectra of reagents used in this work are presented in Figures 2 and 3. Poly(lactic acid) spectrum contains characteristic absorption bands at 2800-3000, 1300-1500 and $756 \mathrm{~cm}^{-1}$ which can be assigned to methylene groups (stretching and defor- 

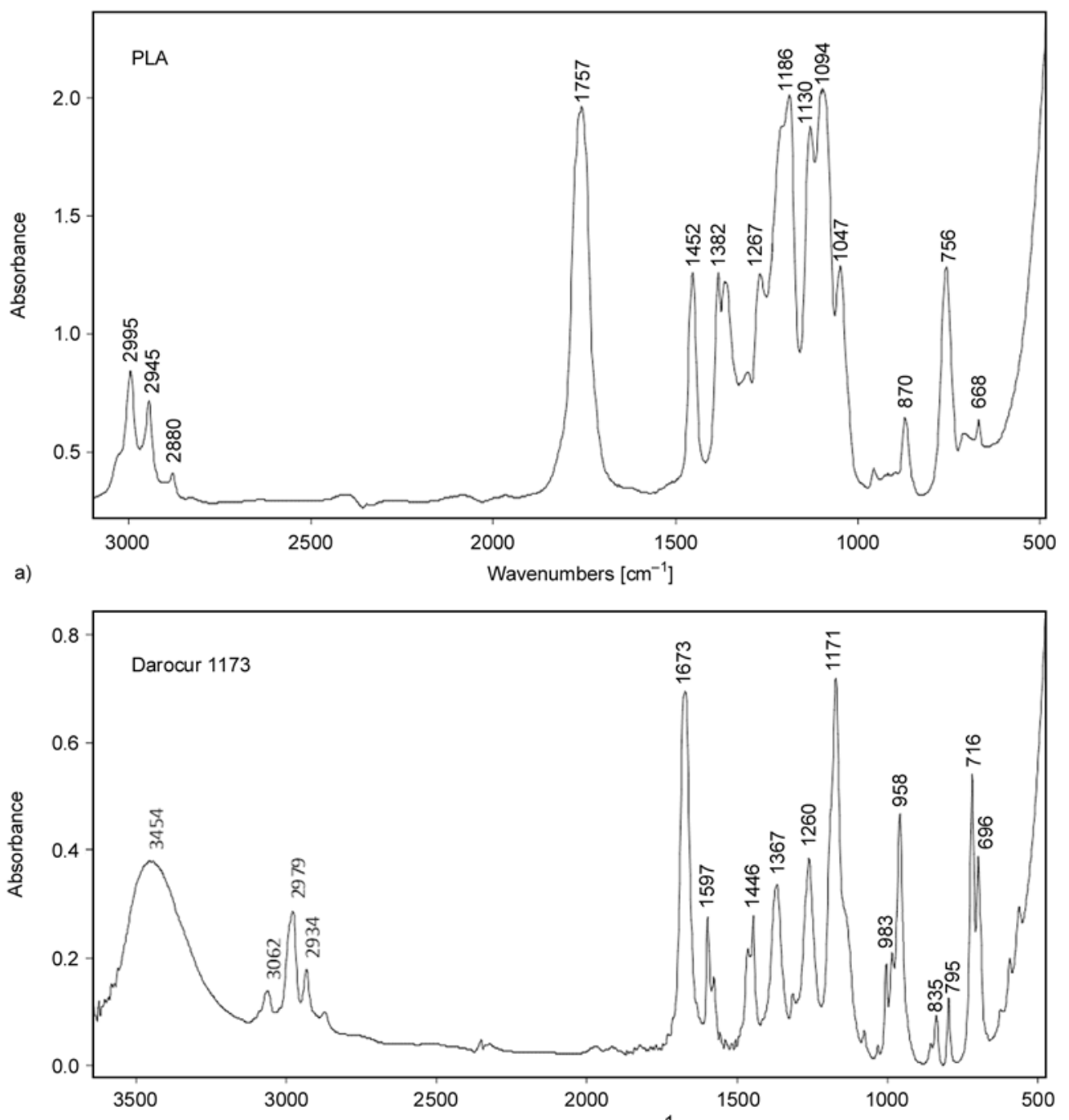

b)

Wavenumbers $\left[\mathrm{cm}^{-1}\right]$

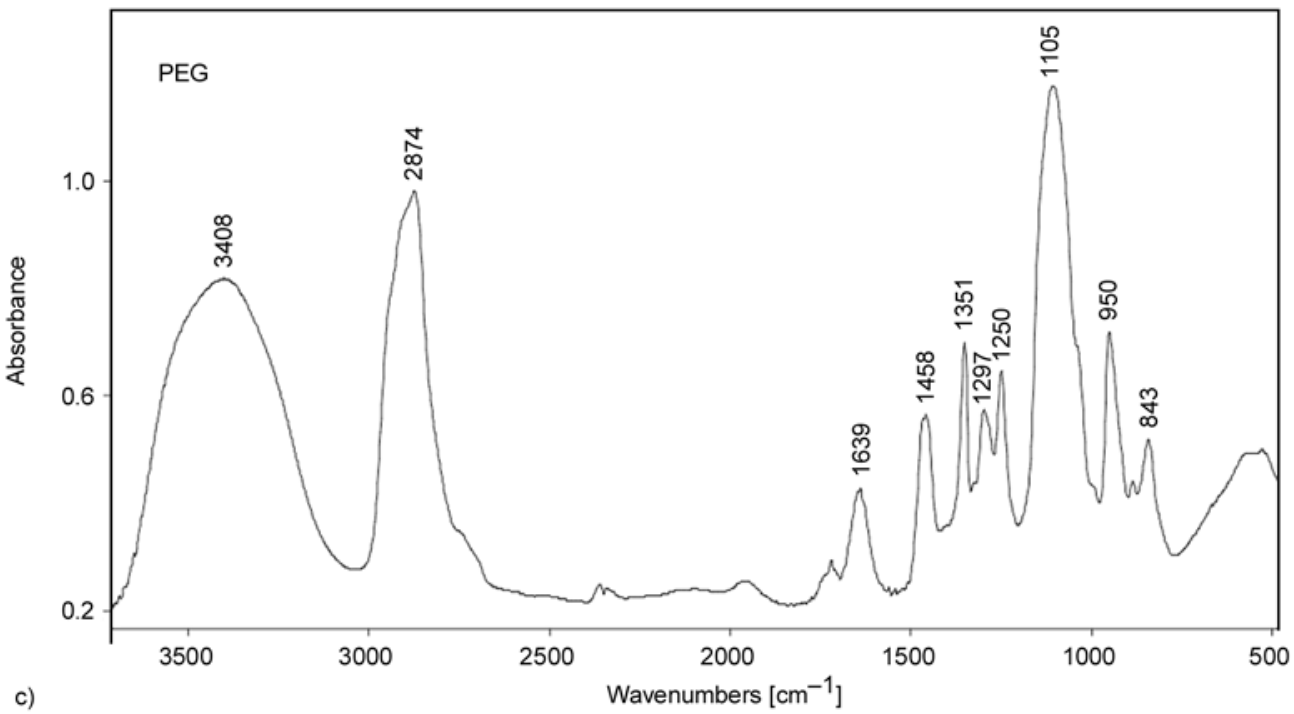

Figure 2. FTIR spectra of virgin reagents: PLA (a), photoinitiator - Darocur 1173 (b) and plasticizer - PEG (c)

mation vibrations, respectively). Strong, sharp ure 2a). Only very small, residual hydroxyl band at peaks at $1757 \mathrm{~cm}^{-1}$ due to carbonyls and $1186, \sim 3500 \mathrm{~cm}^{-1}$ can be seen. $1094 \mathrm{~cm}^{-1}$ attributed to $\mathrm{C}-\mathrm{O}-\mathrm{C}$ groups appear (Fig- 
Photoinitiator (Figure 2b) spectrum exhibits intensive hydroxyl absorption (with maximum at $3460 \mathrm{~cm}^{-1}$ ), bands at 2800-3100 range, at 1597, 1446, 1367, $958,716 \mathrm{~cm}^{-1}$ assigned to aliphatic and aromatic $\mathrm{C}-\mathrm{H}$ (stretching and bending), $1673 \mathrm{~cm}^{-1}$ (carbonyl) and $1171 \mathrm{~cm}^{-1}(\mathrm{C}-\mathrm{O}-\mathrm{C}$ vibrations).

Plasticizer (PEG) spectrum is relatively simple: broad hydroxyl band is centered at about $3400 \mathrm{~cm}^{-1}$, methylene at $2874,1458,1351,1250,950$ and $843 \mathrm{~cm}^{-1}$ (stretching and deformation) and ether (strong) at $1113 \mathrm{~cm}^{-1}$ (Figure 2c). Additionally, two low intensity peaks appear at carbonyl region $\left(1721,1639 \mathrm{~cm}^{-1}\right)$ indicating some internal impurities.

As expected, the spectra of tri- tetra- and pentacrylate monomers are very similar because they con- tain the same type of functional groups (Figure 3). The methyl/methylene (2800-3000 $\mathrm{cm}^{-1}$ stretching region), carbonyl $\left(1726 \mathrm{~cm}^{-1}\right)$ and ether $(1000-$ $1200 \mathrm{~cm}^{-1}$ ) bands exist in all acrylate spectra. The broad hydroxyl band (3300-3700 $\left.\mathrm{cm}^{-1}\right)$ is also seen in all spectra, although $\mathrm{OH}$ groups are not originally present in PETeA structure. The other characteristic bands, appearing at $1634,1468,985$ and $809 \mathrm{~cm}^{-1}$, can be assigned to $\mathrm{C}=\mathrm{C}$ stretching, $\mathrm{C}-\mathrm{H}$ deformation (in-plane), $\mathrm{C}-\mathrm{H}$ deformation (out-of plane) and $=\mathrm{C}-\mathrm{H}$ bending vibrations, respectively.

The distinct difference appears in the stretching methylene range: the ratio of intensities of 2962 to $2898 \mathrm{~cm}^{-1}$ bands $\left(\mathrm{A}_{2962}: \mathrm{A}_{2898}\right)$ decreases with an increase of functional groups in monomer molecule (Figure 3a). It changes in the following order:
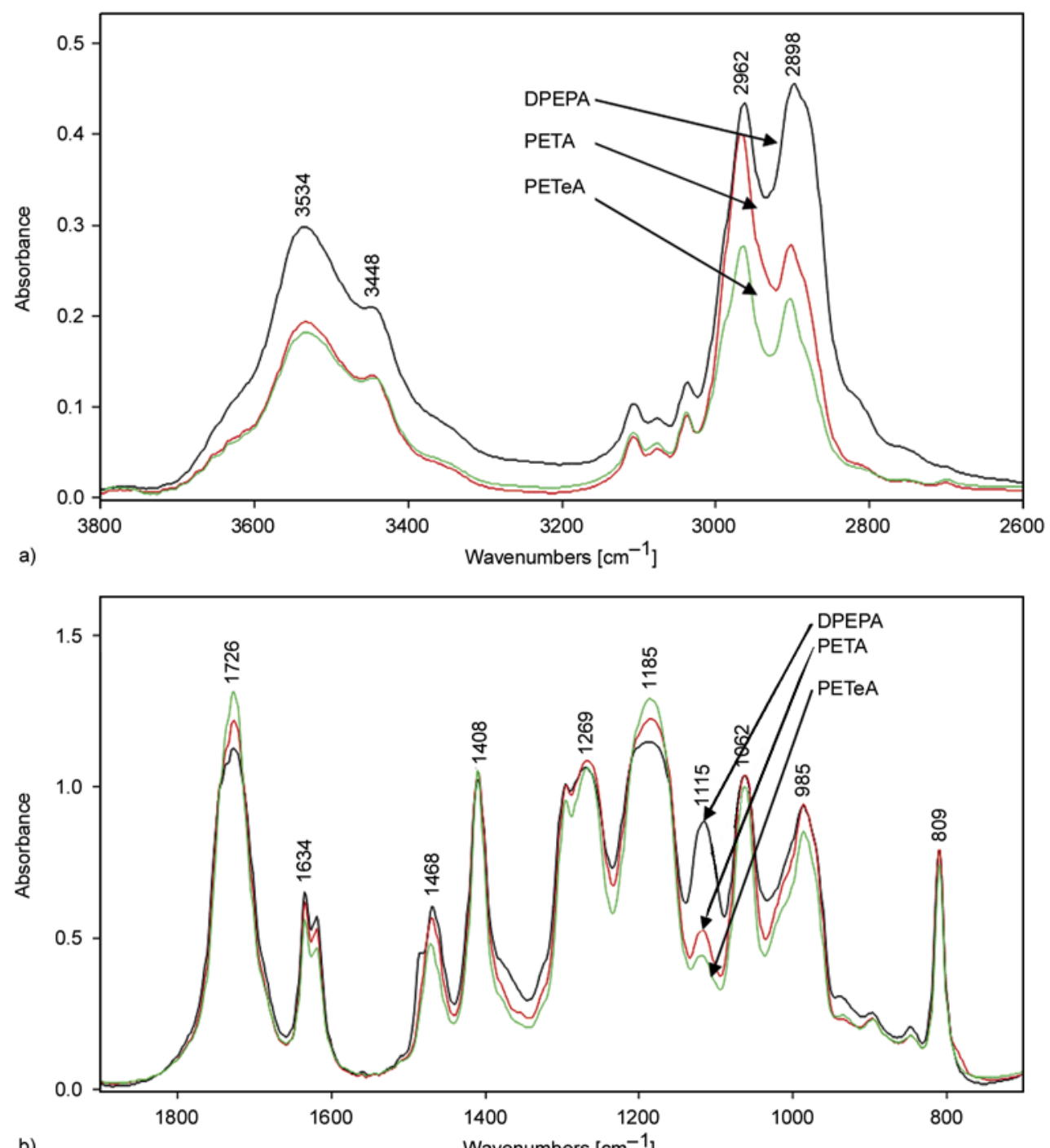

Figure 3. FTIR spectra of three acrylate monomers in $2600-3800 \mathrm{~cm}^{-1}$ (a) and $700-1900 \mathrm{~cm}^{-1}$ (b) ranges (DPEPA - black, PETA - red, PETeA - green) 
$\left(\mathrm{A}_{2962}: \mathrm{A}_{2898}\right)(\mathrm{PETA})=1.452>$

$\left(\mathrm{A}_{2962}: \mathrm{A}_{2898}\right)(\mathrm{PETeA})=1.279>$

$\left(\mathrm{A}_{2962}: \mathrm{A}_{2898}\right)(\mathrm{DPEPA})=0.976$

The second modification is seen in $1115 \mathrm{~cm}^{-1}$ band attributed to ether type vibrations - the highest intensity of this band is observed for DPEPA containing six $\mathrm{C}-\mathrm{O}-\mathrm{C}$ groups in molecule, contrary to PETeA and PETA (four $\mathrm{C}-\mathrm{O}-$ bonds/molecule).

The band at $809 \mathrm{~cm}^{-1}$, due to $=\mathrm{C}-\mathrm{H}$ bending vibrations has been selected for monitoring the progress of photopolymerization.

\subsection{General observations concerning IPN preparation}

The solutions of acrylate/PLA and acrylate/PLA/ PEG blends (containing also photoinitiator) in chloroform as well as solid films obtained after solvent evaporation were completely transparent.

The photopolymerization in all studied systems started immediately after exposure to UV. Process underwent with high efficiency in a few minutes. We decided to finish the kinetic studies after 5 minutes of UV-irradiation although the conversion of double bond was not completed. Longer exposure could be dangerous for IPN specimens because of possibility of photodegradation process. The application of photographic shutter allowed the observation of subtle changes in the first period of photopolymerization and distinguishing the variation between the PLA containing different monomers. The obtained samples of acrylates cured in PLA matrix were translucent, rigid and very brittle, thus poly(ethylene glycol), PEG, was added for plasticization. The course of monomers photopolymerization in the presence of PEG has been also determined.

Figure 4 shows FTIR spectra of acrylate+PLA blends before (black lines) and after 5 minutes UVirradiation (red curves). In all cases the bands characteristic for both components exists. As predicted, the main changes after photopolymerization occur at bands attributed to double bonds in acrylate monomers: at 1635, 1409, 986 and $809 \mathrm{~cm}^{-1}$. The differences in other absorption bands caused by photocuring are negligible. No new additional peak which could indicate that any other reactions take place.
The mechanism of photopolymerization of multifunctional monomers is described as a free-radical chain reaction $[10,12,15]$. During the first stage of process, photosensitive initiator absorbs quanta of radiation and undergoes excitation followed by the creation of initiating species, usually free radicals. The initiation is mainly dependent on the photoinitiator absorption coefficient, the quantum yield of radicals formation and the intensity of radiation.

The next step is propagation, when active radicals react with monomer molecules leading to chain growth. Because the multifunctional monomers contain a few functional groups, the macromolecules become highly branched, moreover, a high rate of reaction is characteristic for such systems. Particularly, the first period of polymerization is fast, then process slows down due to crosslinking and vitrification. Formed network contains a significant amount of trapped or 'frozen' radicals and unreacted double bonds. The probability of their further reaction is limited (because of segmental mobility restrictions) but is possible. The final conversion is strongly dependent on the mobility of the functional groups, which is also determined by the slow relaxation processes occurring at room temperature even in the dark. The termination is a radical recombination or diffusion-controlled reaction.

The differences in the kinetics and efficiency of the acrylates photopolymerization in PLA in this study are connected mainly with different structure and reactivity of monomers because the conditions of curing were kept constant. The monomers differ mainly in their functionality (expressed as the amount of functional groups per molecule or as the number of double bounds per kilogram of monomer) and branching degree. As the viscosity of monomers is very high, the inhibiting action of oxygen is reduced because of its negligible diffusion.

\subsection{Kinetics of polymerization of acrylate monomers alone and in PLA matrix}

The rate of polymerization was evaluated by FTIR spectroscopy on the basis of the band at $809 \mathrm{~cm}^{-1}$, which is attributed to the double bond vibrations in acrylate monomer. Examples of absorbance decrease in FTIR spectrum of photopolymerized PETeA and PETeA in PLA film are shown in Figure 5. Analysis of other bands attributed to unsaturated groups (e.g. $1634,1409 \mathrm{~cm}^{-1}$ ) leads to the same conclusions. 


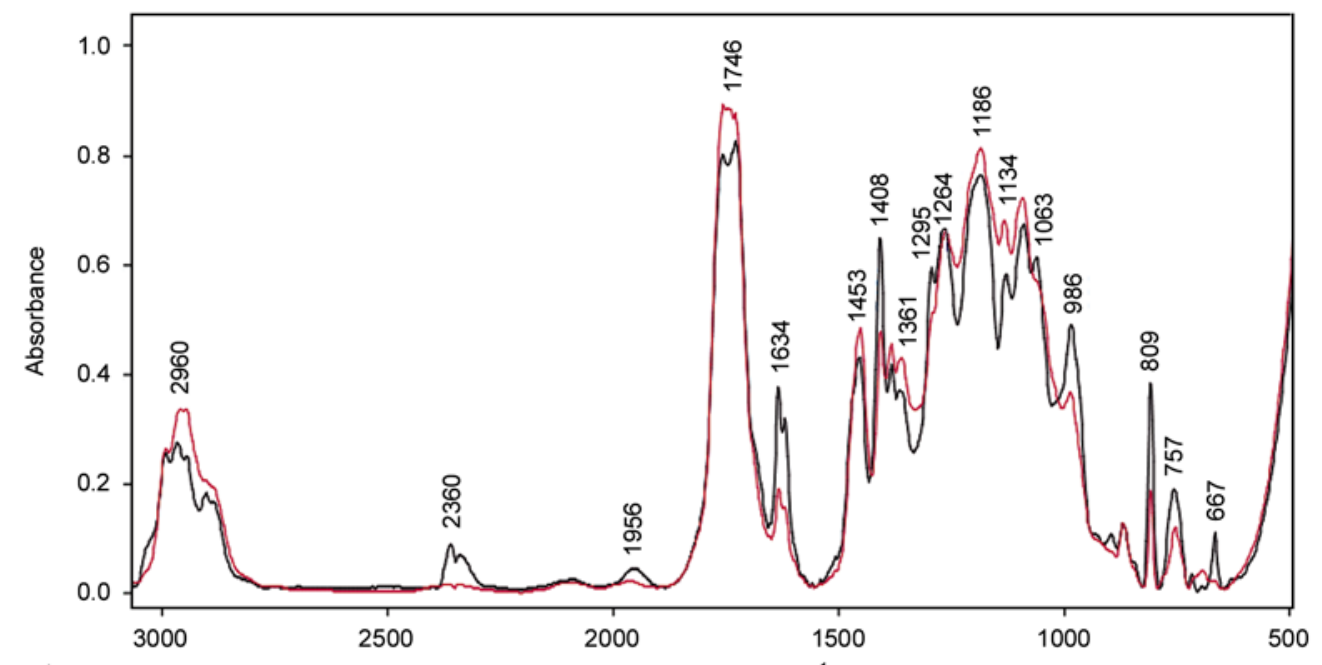

a)

Wavenumbers $\left[\mathrm{cm}^{-1}\right]$

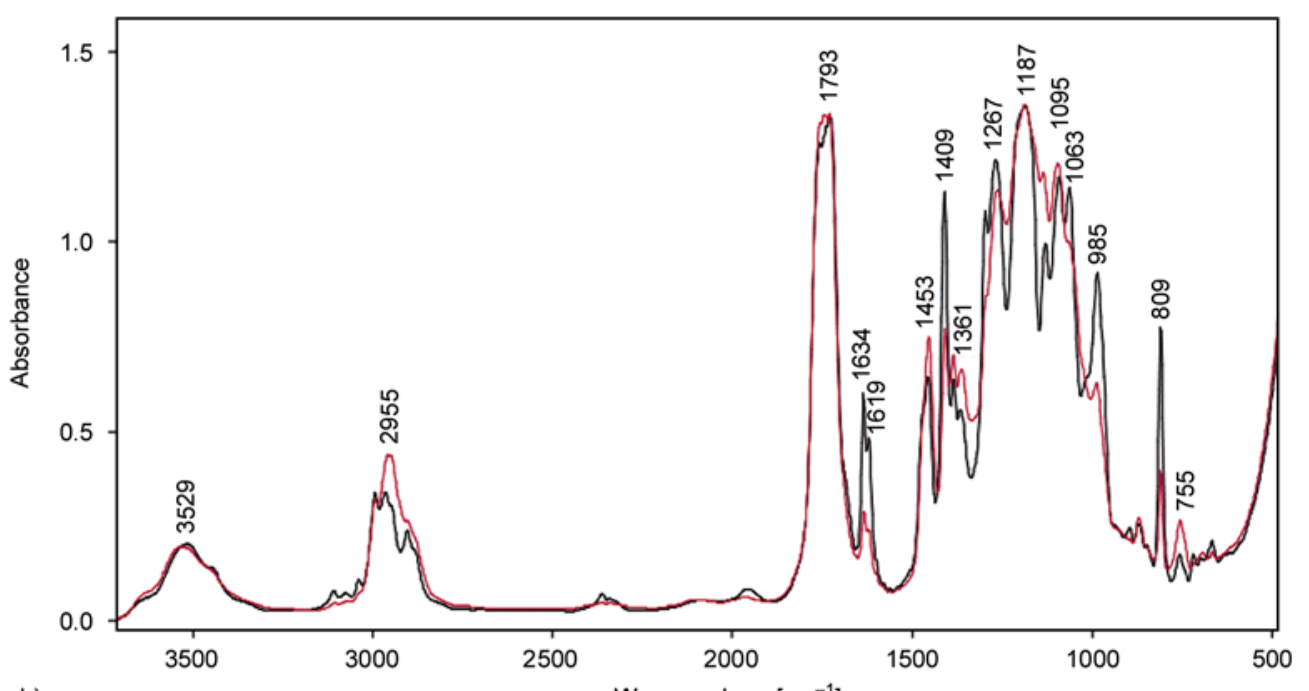

b)

Wavenumbers $\left[\mathrm{cm}^{-1}\right]$

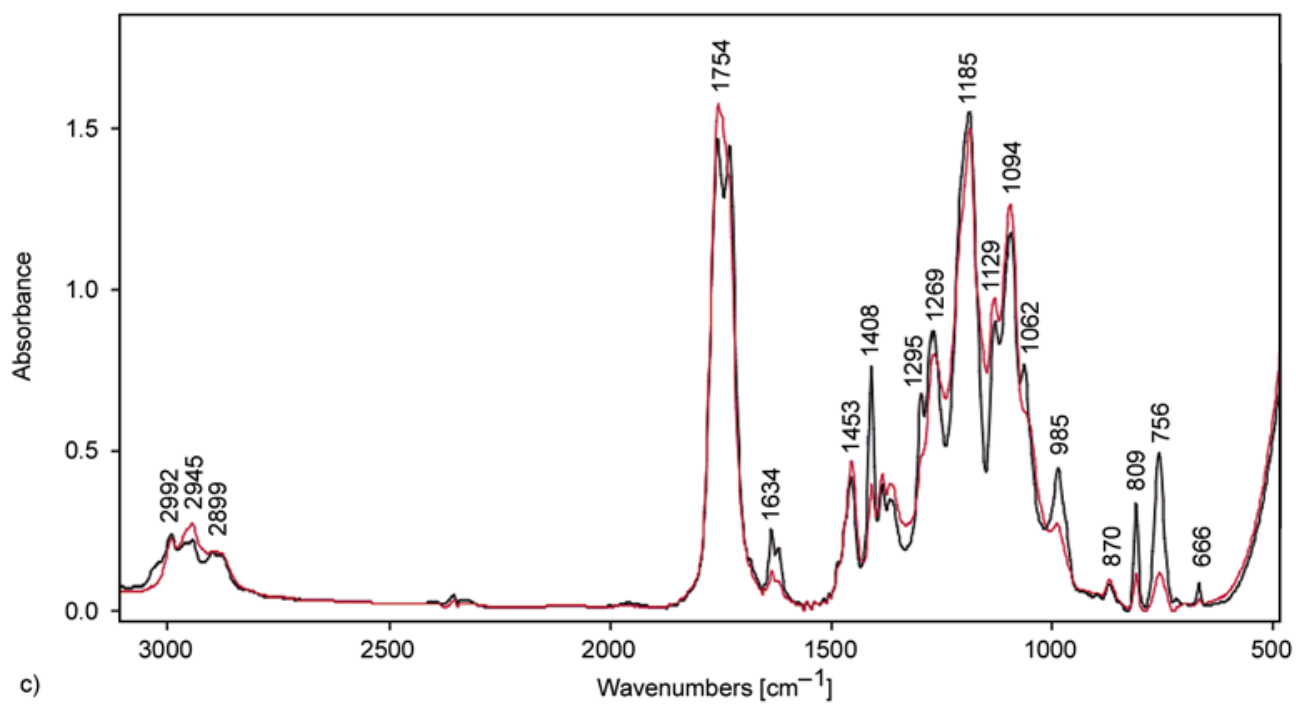

Figure 4. FTIR spectra of acrylate/PLA networks before irradiation (black) and obtained after 5 min photopolymerization (red); PLA/PETA (a), PLA/DPEPA (b) and PLA/ PETeA (c)

Because of the lack of induction period, the inflection point has not been observed on the plot of con- version degree versus exposure time (Figure 6), thus, the maximum polymerization rate, $R_{\mathrm{p}}$ max , was 

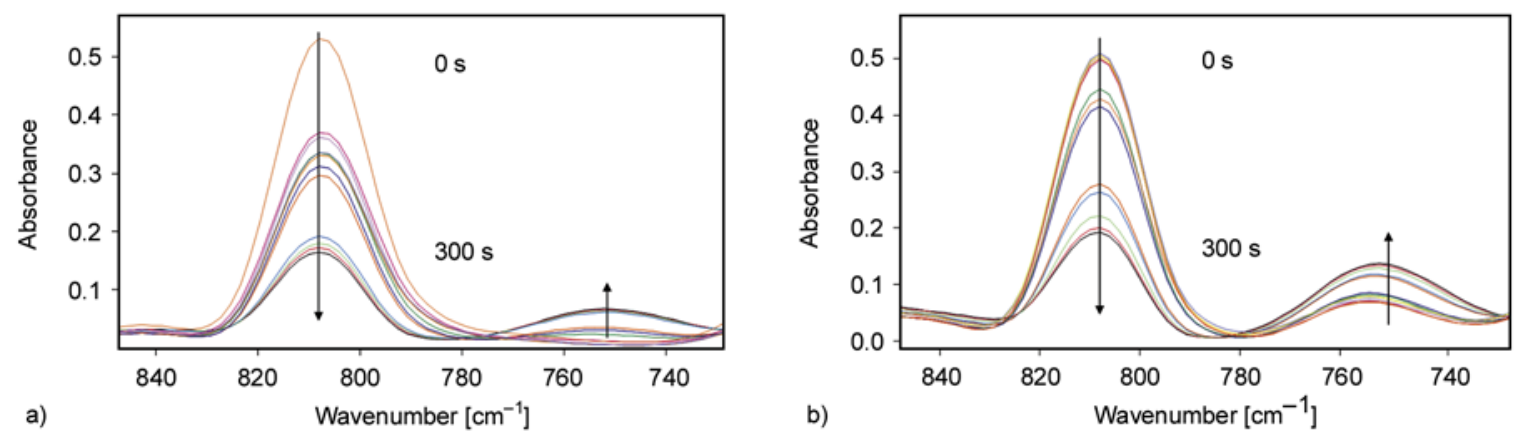

Figure 5. Changes of $\mathrm{C}=\mathrm{C}$ band during 5 min photopolymertization of PETeA (a) and PETeA in PLA matrix (b)

obtained from the slope of straight-line part of these kinetic curves for the beginning period of reaction. Photopolymerization of tri-, tetra- or penta-acrylate monomers (Figure 6a) as well as photocuring of these reagents in PLA matrix, occurs rapidly (Figures $6 \mathrm{~b}$ and $6 \mathrm{c})$. The shapes of conversion curves are similar for DPEPA, PETA and PETeA.

In the case of polymerization of monomers alone, the highest polymerization rate has been observed up to approximately 10 seconds (Figure 6a). At this time the conversion degree achieved already $50 \%$ but the final conversion (after $5 \mathrm{~min}$ of exposure) does not exceed $80 \%$. The course of photopolymerization is similar and kinetic curves partially overlap.

The main kinetic parameters are listed in Table 1 . As one can see, $R_{\mathrm{p}}{ }^{\text {max }}$ slightly decreases with the increase of functional groups for monomers photopolymerized alone, thus, $50 \%$ conversion is reached fastest in PETA (after $2 \mathrm{~s}$ ) and lowest in DPEPA (13 s). For that reason, needed dose for $50 \%$ conversion is relatively low (below $220 \mathrm{~J} / \mathrm{m}^{2}$ ). After approximately 50 seconds, the polymerization significantly slows down and finally, after about 2 minutes of exposure, a plateau is observed. Such behavior is in accordance to results obtained previously for these monomers in similar conditions $[11,16]$.

Photopolymerization rates of acrylates in PLA films (Figure 6b) are lower than those previously observed without polymeric matrix. Unexpectedly, the highest $R_{\mathrm{p}}{ }^{\text {max }}$ among these three specimens was observed for PETeA in PLA. The maximum rate can be ordered as follows:

$R_{\mathrm{p}}{ }^{\max }(\mathrm{PETe} \mathrm{A}+\mathrm{PLA})>R_{\mathrm{p}}{ }^{\max }(\mathrm{DPEPA}+\mathrm{PLA})>$ $R_{\mathrm{p}}{ }^{\max }(\mathrm{PETA}+\mathrm{PLA})$
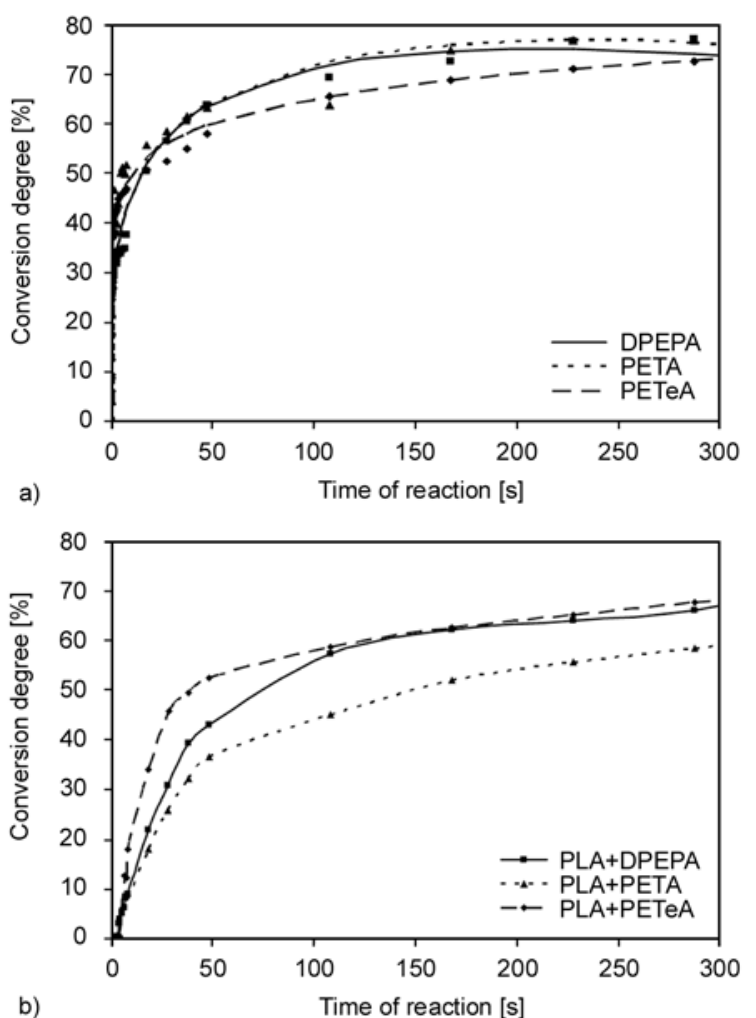

b)

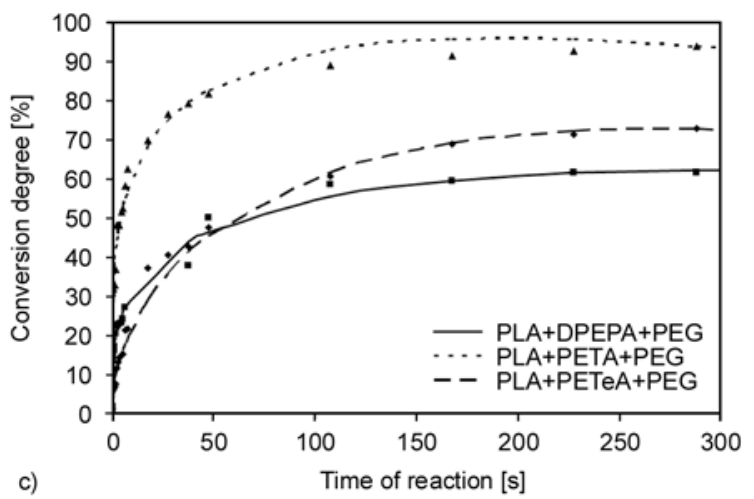

Figure 6. Kinetics of photopolymerization of acrylates (a), acrylates in PLA matrix (b) and acrylate/PLA/ PEG formulations (c); presence of photoinitiator $-5 \mathrm{wt} \%$, process conducted in air atmosphere at room temperature upon a HPK lamp 
Since, the $50 \%$ conversion degree in these formulations is reached after longer irradiation time (comparing to $\sim 10 \mathrm{~s}$ for pure monomers) i.e. at $\sim 40,70$ and $150 \mathrm{~s}$ in PETeA + PLA, DPEPA + PLA and PETA + PLA, respectively. Therefore, the energy necessary for fifty percentage conversion is high (ca. $650-2580 \mathrm{~J} / \mathrm{m}^{2}$ ). The final efficiency is also lower for all monomers in the blends (below $70 \%$ for DPEPA + PLA or PETeA + PLA and about $60 \%$ for PETA + PLA). It clearly indicates that acrylate photopolymerization is hampered in the PLA network, which is obviously caused by the trapping of growing macroradicals in polymer matrix, which is in glassy state in the room temperature.

Plasticization of PLA by PEG causes an increase of polymerization rate in the first step compared to the

Table 1. The parameters characterizing the kinetics of polymerization of acrylate monomers (alone), acrylate monomers in PLA and acrylate + PLA + PEG ternary systems obtained from FTIR

\begin{tabular}{|c|c|c|c|c|}
\hline Sample & $\begin{array}{c}\mathbf{R}_{\mathbf{p}}{ }^{\max } \\
{\left[\% \cdot \mathrm{s}^{-1}\right]}\end{array}$ & $\begin{array}{c}X(5 ') \\
{[\%]}\end{array}$ & $\begin{array}{c}\mathbf{t}(50 \%) \\
{[\mathbf{s}]}\end{array}$ & $\begin{array}{c}\mathrm{D}(\mathbf{5 0 \%}) \\
{\left[\mathrm{J} / \mathrm{m}^{2}\right]}\end{array}$ \\
\hline PETA & 2.7 & 77 & 2 & 33 \\
\hline PETeA & 2.3 & 73 & 10 & 167 \\
\hline DPEPA & 1.6 & 74 & 13 & 216 \\
\hline PETA + PLA & 1.0 & 59 & 155 & 2581 \\
\hline PETeA + PLA & 2.1 & 68 & 39 & 649 \\
\hline DPEPA + PLA & 1.3 & 67 & 72 & 1199 \\
\hline PETA + PLA + PEG & 10.0 & 94 & 5 & 83 \\
\hline PETeA + PLA + PEG & 3.0 & 73 & 62 & 1032 \\
\hline DPEPA + PLA + PEG & 7.7 & 62 & 69 & 1149 \\
\hline
\end{tabular}

$R_{\mathrm{p}}{ }^{\text {max }}$ - maximum polymerization rate; $\mathrm{X}\left(5^{\prime}\right)$ - final conversion degree after 5 min UV action, $t(50 \%)$ - time needed for $50 \%$ conversion; $D(50 \%)$ - dose needed for $50 \%$ conversion of monomer. Formulations and reaction conditions: acrylate to PLA ratio $=1: 1$, $5 \%(\mathrm{~m} / \mathrm{m})$ Darocur $1173,10 \%(\mathrm{~m} / \mathrm{m})$ PEG placticizer, UV-source HPK lamp, incident intensity $-16.65 \mathrm{~W} / \mathrm{m}^{2}$, air atmosphere and room temperature. course of reaction in nonplasticized PLA. $R_{\mathrm{p}}{ }^{\max }$ values are even higher than those for monomers cured alone (Table 1). 50\% conversion is achieved after about 5, 60 and $70 \mathrm{~s}$ of UV action in PETA + PLA + PEG, PETeA+PLA+PEG and DPEPA + PLA + PEG, respectively. Assuming that polymer plasticization facilitates the polymerization process because of higher mobility of acrylate monomers and radicals in PLA blends, we can conclude that the best plasticizing effect was found in the network containing PETA. The formation of IPN in this formulation is saving-energy process $\left(83 \mathrm{~J} / \mathrm{m}^{2}\right)$.

The order of $R_{\mathrm{p}}{ }^{\max }$ in ternary blends is reversed comparing to the previously indicated for nonplasticized systems:

$$
\begin{aligned}
& R_{\mathrm{p}}{ }^{\max }(\mathrm{PETA}+\mathrm{PLA}+\mathrm{PEG})> \\
& R_{\mathrm{p}}{ }^{\max }(\mathrm{DPEPA}+\mathrm{PLA}+\mathrm{PEG})> \\
& R_{\mathrm{p}}{ }^{\max }(\mathrm{PETe}+\mathrm{PLA}+\mathrm{PEG})
\end{aligned}
$$

It means that plasticizer increases the rate of phtotopolymerization and this effect is particularly high in the case of PETA + PLA + PEG system where additionally the great conversion degree (exceeding 90\%) was found (Figure 6c).

The conversion degree is strictly connected to the number of unreacted acrylate bonds in formed IPN (Table 2), which is the largest in the case of PETA + PLA (nonplasticized) and DPEPA + PLA + PEG.

However, the amount of unsaturations can be reduced by the heating of interpenetrating network above glass transition temperature in which post-polymerization takes place owing to improvement of macrochain mobility and reactivity [17].

Table 2. The amount of gel [\%] formed in acrylate monomers, acrylate monomer + PLA blends and acrylate + PLA + PEG,

\begin{tabular}{|c|c|c|c|c|}
\hline Sample & $\begin{array}{c}\text { Total amount of gel } \\
{[\%]}\end{array}$ & $\begin{array}{c}\text { Gel containing PLA } \\
{[\%]}\end{array}$ & $\begin{array}{c}\text { Degree of PLA grafting } \\
{[\%]}\end{array}$ & $\begin{array}{c}\text { Number of unreacted } \mathrm{C}=\mathrm{C} \\
{[\%]}\end{array}$ \\
\hline PETA & 99.5 & - & - & 23 \\
\hline PETeA & 99.7 & - & - & 27 \\
\hline DPEPA & 100.0 & - & - & 26 \\
\hline PETA + PLA & 96.6 & 46.6 & 93.2 & 41 \\
\hline PETeA + PLA & 94.4 & 44.4 & 88.8 & 32 \\
\hline DPEPA + PLA & 88.8 & 38.8 & 77.6 & 33 \\
\hline PETA + PLA + PEG & 93.3 & 43.3 & 86.6 & 6 \\
\hline PETeA + PLA + PEG & 98.8 & 48.8 & 97.6 & 27 \\
\hline DPEPA + PLA + PEG & 74.8 & 24.8 & 49.6 & 48 \\
\hline
\end{tabular}
estimated grafting degree of PLA and number of unreacted double bonds after 5 minutes of photoreaction (conditions as in Table 1). 


\subsection{Gel formation of pure acrylate monomers and in PLA matrix}

The acrylate monomers applied for preparation IPN are known as crosslinking agents. In fact, the efficiency of gel formation in our system is very high. An insoluble gel was formed in both cases: during photopolymerization of acrylate monomer alone as well as in PLA matrix (also in the presence of plasticizer). The values of percentage amount of gel after 5 min photocuring are listed in Table 2 .

One can observe, that gel content exceeds $50 \%$ in all polyacrylate/PLA specimens (although the amount of introduced monomer is always $50 \%$ ). It clearly indicates that PLA, which does not undergo photocrosslinking when is exposed alone, has to participate in photoprocesses in the presence of acrylate monomers. Two possibilities should be taken into consideration: first, most probable, is physical entanglement of PLA macrochains in the rapidly formed acrylate system. This way interpenetrating polymer network is formed. Second option is photografting process of increasing acrylate molecule onto PLA chains. It suggests that created free radicals (from initiator photolysis or growing acrylate macroradicals) are able to abstract hydrogen atom from PLA. The simple recombination of two types of macroradicals (PLA and polyacrylate) forms the graft or random copolymer. Direct grafting is also probable because HPK lamp emits high energy radiation and some free macroradicals can be generated in PLA, which can further react with acrylate monomer, recombine with any other (macro)radical or undergo chain transfer reaction (onto polyacrylate). Another possibility of formation of covalent bonds between both network constituents is possibility of ester exchange (transesterification) between ester PLA groups and hydroxyl groups present in PETA and DPEPA:

$$
\mathrm{RCOOR}^{\prime}+\mathrm{R}^{\prime \prime} \mathrm{OH} \rightleftharpoons \mathrm{RCOOR}{ }^{\prime \prime}+\mathrm{R}^{\prime} \mathrm{OH}
$$

where RCOOR' is PLA and $\mathrm{R}^{\prime \prime} \mathrm{OH}$ is PETA, DPEPA or polyPETA, polyDPEPA.

Transesterification is also possible between PLA macromolecules and PEG which contain hydroxyl end-groups. Moreover, monomers containing $\mathrm{OH}$ groups (PETA and DPEPA) can directly participate in the additional curing with PEG and PLA.

Assuming that the monomer in the blend was completely crosslinked, one can conclude that the graft- ing degree, expressed as the amount of PLA involved in this process, is very high (Table 2). The highest amount of gel was found in PETA + PLA (96.6\%), which allows determining the grafting degree of PLA equal to $93.2 \%$. Both remained samples, are characterized by slightly lower PLA grafting degree: 88.8 and $77.6 \%$ in PETeA + PLA and DPEPA+ PLA, respectively. One can conclude that the higher functionality of acrylate monomer negligibly hampers the efficiency of photografting onto PLA, which is probably caused by the competition of very efficient crosslinking of multifunctional monomer. The added plasticizer slightly modifies the photocrosslinking process of acrylates/PLA blend. The total amount of gel formed after 5 minutes exposure is continually very high $(\sim 75-99 \%)$, which evidently indicates that besides of acrylates also PLA and PEG molecules are entangled and trapped in IPN.

\subsection{Glass transition temperature $\left(T_{\mathrm{g}}\right)$}

Pure PLA sample exhibits the glass transition at $56^{\circ} \mathrm{C}$. Moreover, DSC curve contains the crystallization peak above $150^{\circ} \mathrm{C}$. Pure polyacrylates (PETA, PETeA and DPEPA) do not show the glass transition under the applied conditions (temperature range: from -50 to $220^{\circ} \mathrm{C}$ ). It can be caused by high crosslinking density in these samples - the crosslinking usually strongly depresses the chain mobility.

In the composition of PLA with polyacrylates, an increase of $T_{\mathrm{g}}$ by about $15-18^{\circ} \mathrm{C}$ is observed. It is an evidence of restricted chain mobility. As was suggested above, PLA macromolecules are entangled or even covalently bound to crosslinked acrylates (resulting of grafting).

PEG is a flexible polymer characterized by very low glass temperature (below $0^{\circ} \mathrm{C}$ ) and develops flexibility to other polymers. The plasticization effect is generally attributed to the increase of free volume facilitating macromolecular movements. However, the introduction of PEG (as plasticizer) to PLA+ polyacrylate blend only slightly decreases the glass transition temperature.

The plasticization of PLA has been the subject of several works [18-21] but the mechanism is still controversial. Blending of polymers containing functional groups induces intermolecular interactions and excess volume formation. Owing to terminal hydroxyl groups, PEG chains can participate in 
Table 3. Glass transition temperature of PLA in studied systems determined from DSC curves

\begin{tabular}{|l|c|}
\hline \multicolumn{1}{|c|}{ Sample } & $\mathbf{T}_{\mathbf{g}}\left[^{\mathbf{0}} \mathbf{C}\right]$ \\
\hline PLA & 56 \\
\hline PETA + PLA & 71 \\
\hline PETeA + PLA & 73 \\
\hline DPEPA + PLA & 74 \\
\hline PETA + PLA + PEG & 68 \\
\hline PETeA + PLA + PEG & 69 \\
\hline DPEPA + PLA + PEG & 67 \\
\hline
\end{tabular}

hydrogen bonding and act as 'flexible crosslinks'. The other model is based on the dissolving the polymer in excess PEG, however, it needs higher plasticizer concentration (in our case it was only $10 \% \mathrm{wt}$ ). Although higher amount of PEG usually enhances polymer plasticization, the opposite effect can be observed when phase separation occurs. In studies by Piotrowska et al. [18] it was described that the phase separation occurred also during the crystallization in poly(L-lactide) plasticized with poly (propylene glycol).

\subsection{Samples morphology}

The microstucture of PLA and its interpenetrating networks with three polyacrylates was observed by means of optical microscopy (Figure 7). Pure PLA forms homogeneous, clear film, typical for one component systems. Only small defects can be observed on PLA microphotographs. In microscopic magnification, crystalline forms of PLA are not seen. Probably, the size of crystalline structures are to small for observation under optical microscope.

The structure of polyacrylate + PLA networks (Figures $7 \mathrm{~b}-\mathrm{e}$ ) is more diversified, which indicates sample heterogeneity, in spite of the homogeneity of solutions used for film preparation. It means that phase separation occurs just after solvent evaporation, however, the clear phase border is not distinguished on microscopic images. In all studied acrylate/PLA films, small inclusions and regions of different morphology are observed. The size of single grains dispersed in PLA is ca. 5-10 $\mu \mathrm{m}$. Larger particles, indicating the possibility of their aggregation, can be also seen, particularly in PETA + PLA sample (Figure $7 b$ ). However, it should be pointed out that the size of phase-separated domains is controlled by the network density determined by the high functionality of acrylates.
Interesting images were found for samples containing additionally PEG as plasticizer, which is a semicrystalline polymer (Figure 7e-h).

The structure of PLA containing PEG (plasticizer) is rather homogeneous (Figure 7e). Only small inclusions are rarely observed. Comparing images of pure PLA (Figure 7a) and PLA + PEG (Figure 7e) one can conclude that just PEG is responsible for these small imperfections.

PEG formed spherulitic structures (more or less regular) in matrix of PLA and its IPN with PETeA and DPEPA (Figures $7 \mathrm{~g}$ and $7 \mathrm{~h}$ ). The size range of these crystallites is from few to $200 \mu \mathrm{m}$. The morphology of PETA + PLA + PEG specimen (Figure $7 \mathrm{f}$ ) is rather dissimilar to both others plasticized networks: protrusions of different diameters (from twenty to above $200 \mu \mathrm{m}$ ) appear. This different morphology partially explains the different behavior of this sample upon UV action.

As was demonstrated (Figure 6c), the highest curing rate and conversion degree was found in this case. It suggests that observed morphological structures are characterized by other molecules arrangement (probably less ordered), consequently the plasticizing effect is more evident. Probably, the hydroxyl groups from PETA and PEG interact, which retards the crystallization of plasticizer. Although $\mathrm{OH}$ groups are also present in DPEPA but this monomer is more branched, therefore, steric hindrances can make difficult hydrogen bonding formation. In consequence, the rate and efficiency of PETA polymerization in PLA + PEG are higher than those for DPEPA in PLA + PEG.

The specific details of samples structure have been also observed by SEM. The chosen examples are shown in Figure 8. Pure PLA and polyacrylates are relatively uniform with exception of PETA+PLA (Figure 8b). An altered, more heterogeneous morphology was found in the case of ternary blends: polyacrylate+PLA+PEG (Figure 8e-h).

It is necessary to remind that photographs in Figure 8 present the surface morphology of the samples, which can be different from internal structure. The surface of samples composed of acrylates, PLA and PEG is not smooth, some protrusions of different sizes are observed. It indicates that under top layer heterogeneous domains (connected to crystallization and phase separation) were formed. 

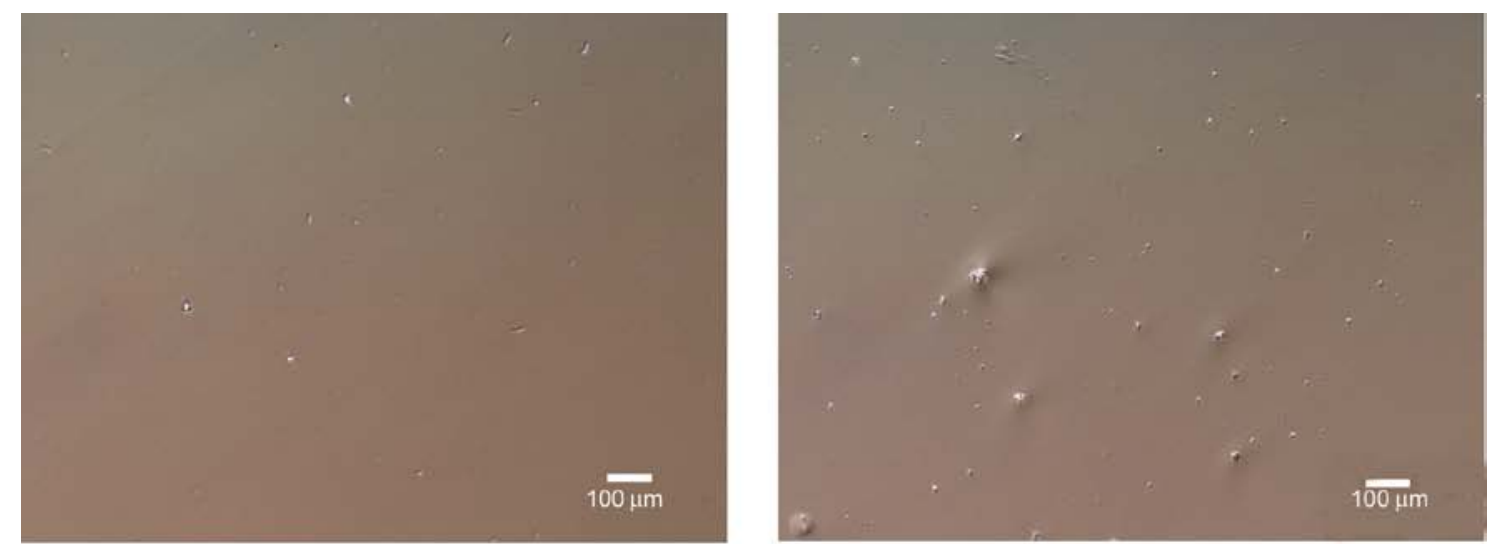

a)

e)
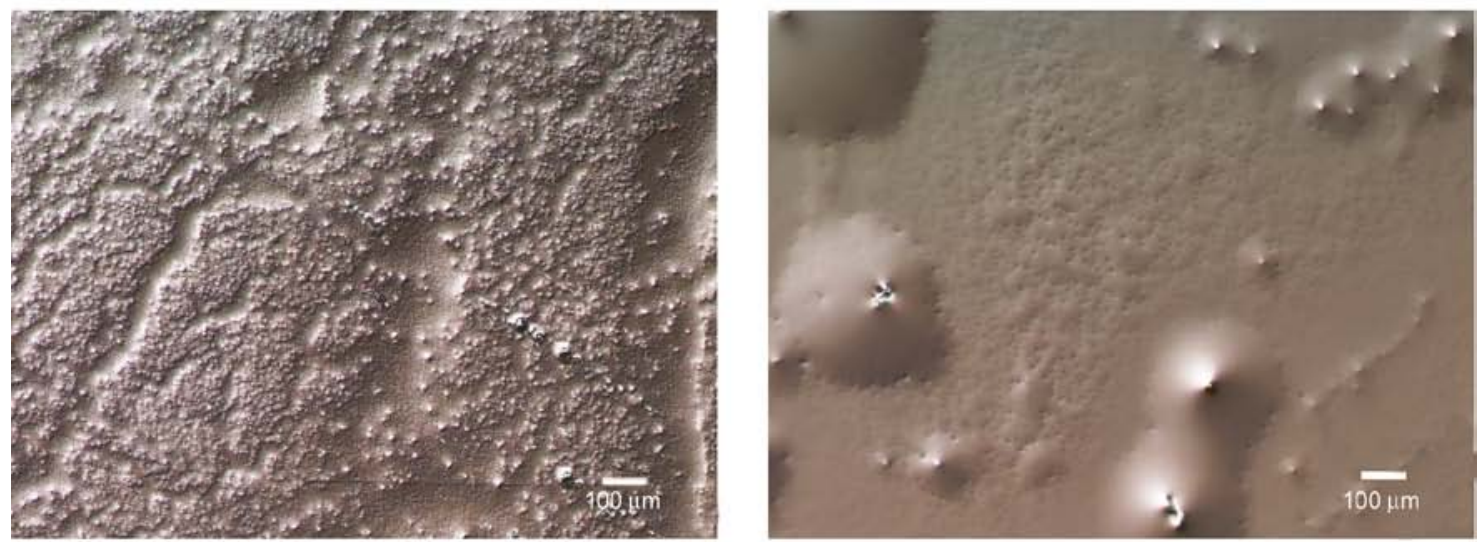

b)

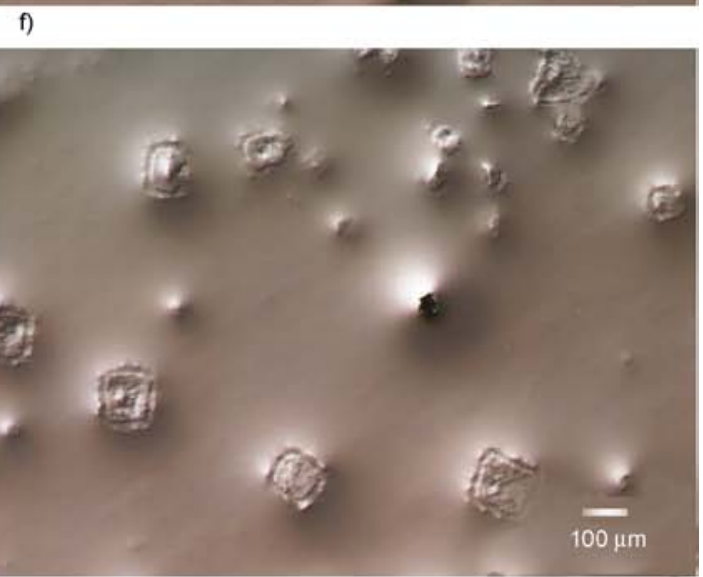

$100 \mu \mathrm{m}$

c)

g)
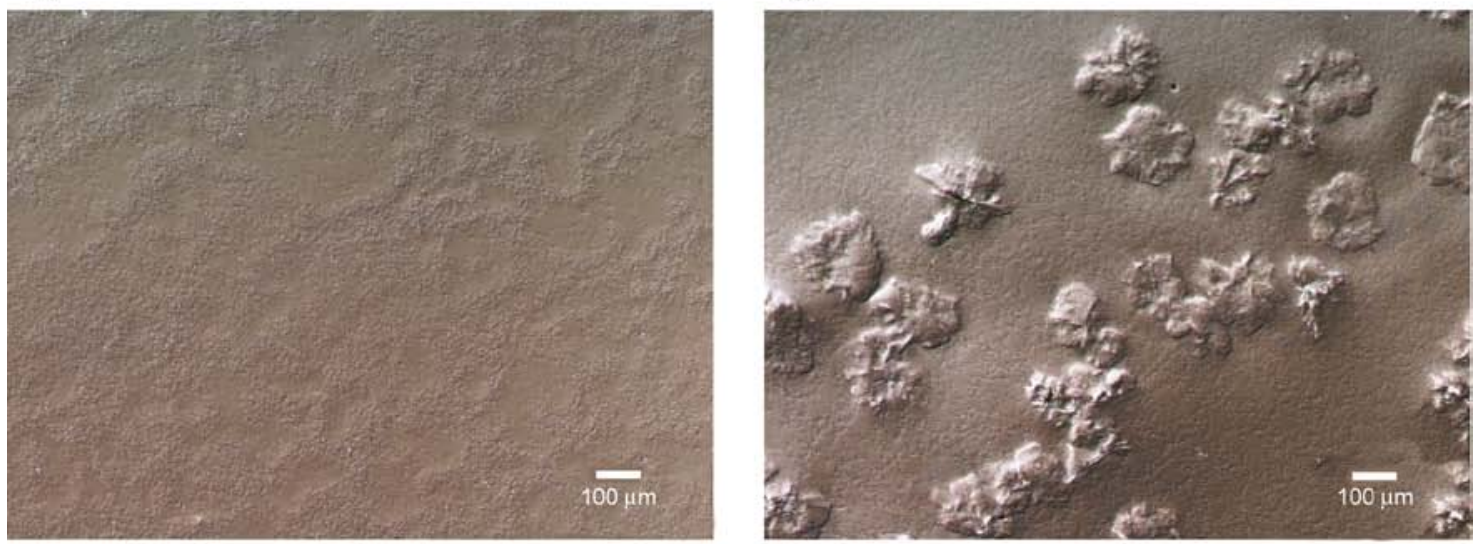

h)

d)

Figure 7. Microphotographs of PLA (a) and photopolymerized blends: PETA + PLA (b), PETeA + PLA (c) and DPEPA+ PLA (d) as well as PLA + PEG (e) and photopolymerized ternary blends: PETA + PLA + PEG (f), PETeA+PLA+ PEG $(\mathrm{g})$ and DPEPA + PLA + PEG (h) 


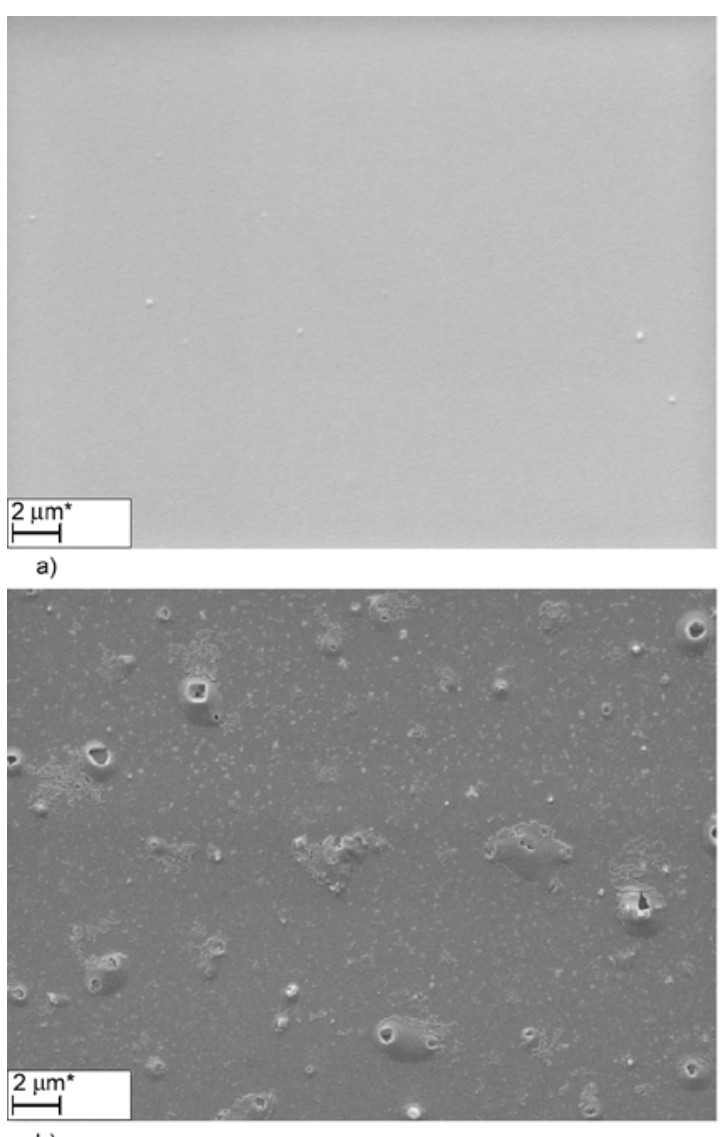

b)

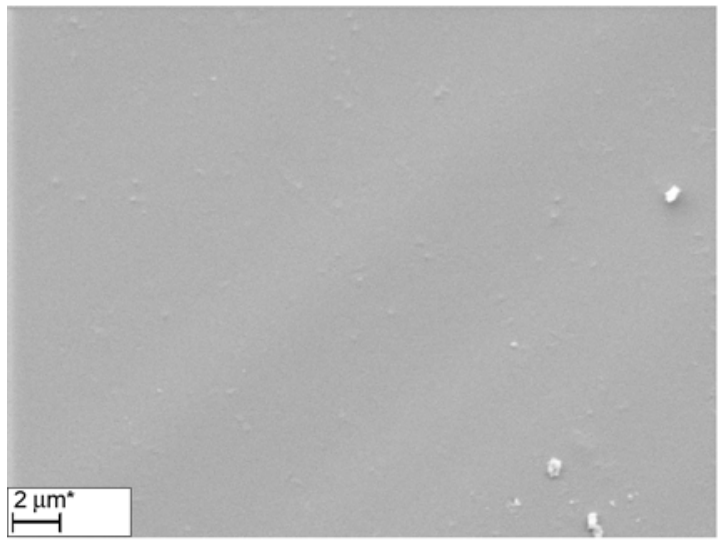

c)

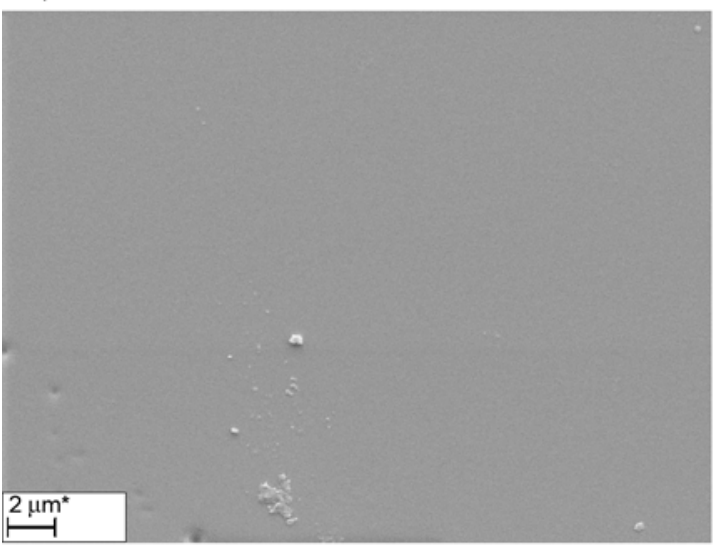

d)

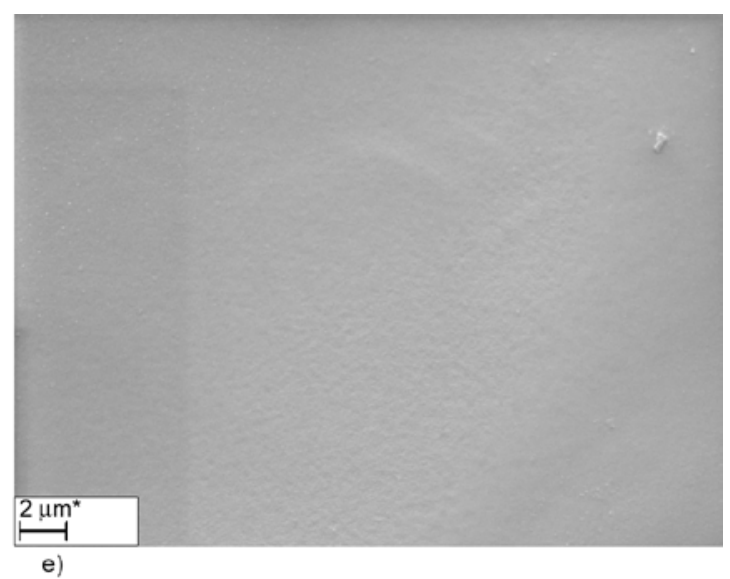

e)
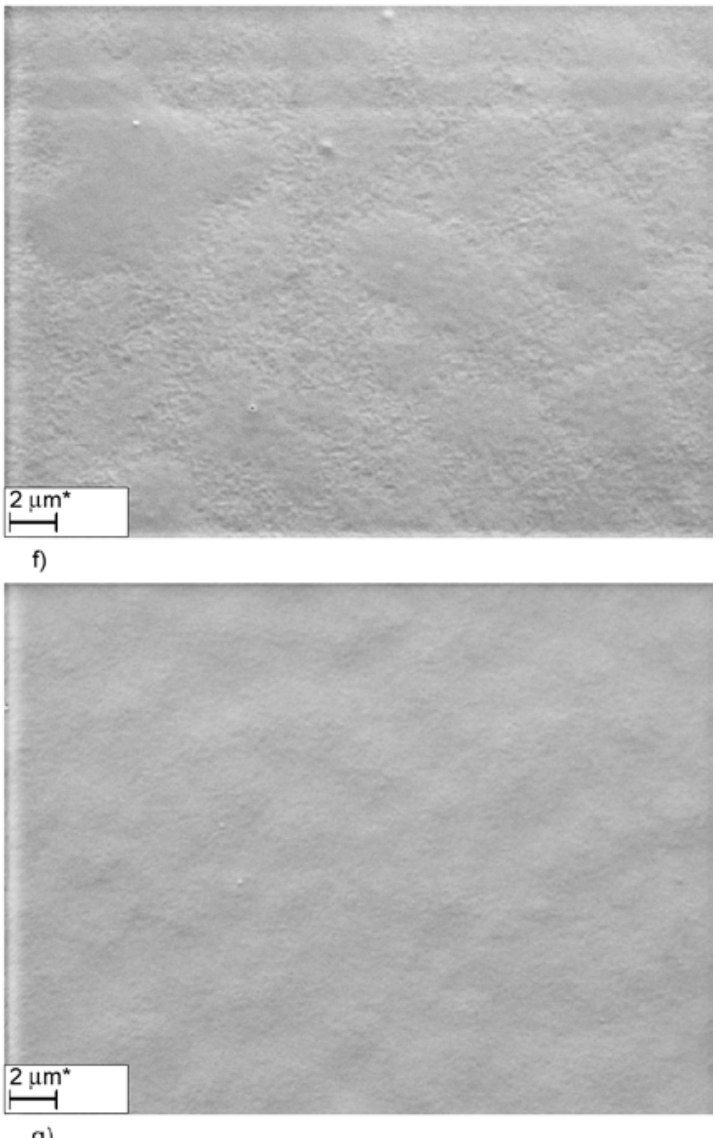

g)

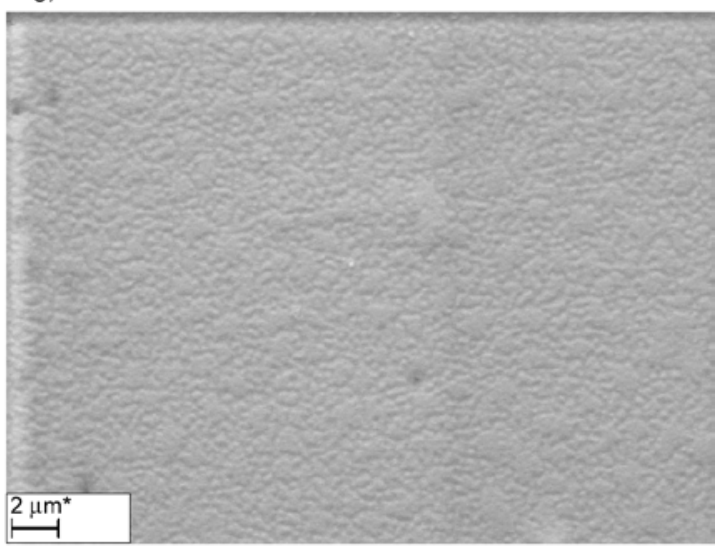

h)

Figure 8. SEM images of PLA (a) and photopolymerized blends: PETA+PLA (b), PETeA + PLA (c) and DPEPA + PLA (d) as well as PLA+PEG (e) and photopolymerized ternary blends: PETA + PLA + PEG (f), PETeA + PLA + PEG (g) and DPEPA + PLA + PEG (h) 
On the other hand, surface morphology determines the useful properties of thin polymeric films applied as packaging materials.

Finally it should be mentioned, that networks morphology strongly depends on the conditions of their preparation. It was observed in the case of three components mixture (acrylate + PLA + PEG) obtained by casting from a common solvent. Slow drying in air atmosphere led to formation of relatively large crystalline structures of PEG in network, while the fast drying in vacuum caused generation of numerous tiny spherulite precursors. Nevertheless, they are often covered by a layer of PLA, which forms a kind of surface 'skin'.

\subsection{Miscibility considerations}

The IPN heterogeneity observed in microphotographs suggests the component separation just after solvent evaporation from PLA/acrylate mixture. The prediction of component miscibility is possible comparing Hildebrand solubility parameters ( $\delta)$ [22].

The literature values of solubility parameters of PLA and poly(methyl acrylate) or poly(methyl methacrylate) [22-24], chosen as a model compounds for polyacrylates obtained from multifunctional monomers, are listed in Table 4.

The differences arising from experimental error (or from different testing method) are rather broad. From cited data, total solubility parameter $\left(\delta_{\text {total }}\right)$ of PLA is $19.28-21.73$ and the $\delta_{\text {total }}$ range for PMA/ PMMA is comparable: $19.1-22.6(\mathrm{~J} / \mathrm{cc})^{0.5}$. According to Hansen's theory, the more detailed analysis can be done using partial solubility parameters concerning dispersion forces $\left(\delta_{\mathrm{d}}\right)$, polar forces $\left(\delta_{\mathrm{p}}\right)$ and hydrogen bonding $\left(\delta_{\mathrm{hb}}\right)[23,24]$. From Table 3 it is seen, that also the partial solubility parameters of PLA and model acrylate polymers have values in a similar range. It suggests that both polymers, thus, the components of our networks, should be miscible according to the theory.

As reported earlier, the photopolymerization of components characterized by a lack or limited miscibility led to the lower conversion degree comparing to compatible mixture $[11,17]$.

On the basis of microscopic studies, we can conclude that phase separation occurs in the mixture of monomer + PLA already during film preparation because of differences in molecular weight of poly-
Table 4. Hansen solubility parameters of PLA, poly(methyl acrylate), PMA, and poly(methyl methacrylate), PMMA from literature data (PMA and PMMA were chosen as a model compounds for studied polyacrylates) [22-24].

\begin{tabular}{|l|c|c|c|c|c|}
\hline Polymer & $\begin{array}{c}\boldsymbol{\delta}_{\mathbf{d}} \\
{\left[(\mathbf{J} / \mathbf{c c})^{\mathbf{0 . 5}]}\right]}\end{array}$ & $\begin{array}{c}\boldsymbol{\delta}_{\mathbf{p}} \\
{\left[(\mathbf{J} / \mathbf{c c})^{\mathbf{0 . 5}}\right]}\end{array}$ & $\begin{array}{c}\boldsymbol{\delta}_{\mathbf{h b}} \\
{\left[(\mathbf{J} / \mathbf{c c})^{\mathbf{0 . 5}}\right]}\end{array}$ & $\begin{array}{c}\boldsymbol{\delta}_{\text {total }} \\
{\left[(\mathbf{J} / \mathbf{c c c})^{\mathbf{0 . 5}}\right]}\end{array}$ & Reference \\
\hline \multirow{4}{*}{ PLA } & 15.80 & 8.7 & 11.10 & 21.10 & {$[23]$} \\
\cline { 2 - 6 } & 17.40 & 7.6 & 10.50 & 21.70 & {$[23]$} \\
\cline { 2 - 6 } & 17.61 & 5.3 & 5.80 & 19.28 & {$[22]$} \\
\cline { 2 - 6 } & 16.85 & 9.0 & 4.05 & 19.53 & {$[22]$} \\
\cline { 2 - 6 } & 18.50 & 9.7 & 6.00 & 21.73 & {$[22]$} \\
\hline PMA & 17.10 & 1.5 & 8.40 & 19.10 & {$[24]$} \\
\hline \multirow{3}{*}{ PMMA } & 18.60 & 10.5 & 7.50 & 22.60 & \multirow{2}{*}[24]{} \\
\cline { 2 - 5 } & 19.10 & 6.5 & 3.90 & 20.50 & \multirow{2}{*}{} \\
\cline { 2 - 5 } & 15.60 & 10.5 & 5.20 & 19.50 & \\
\hline
\end{tabular}

mer and acrylates. Heterogeneity stays after photopolymerization since the NET structure becomes very rapidly inflexible. However, it was proved and explained based on the thermodynamic theory that crosslinking reduces the effective driving force for macrophase separation [25].

On the other hand, the lack of full miscibility is not inconvenient - as it has been reported, the good physical properties of IPN can be obtained from heterogeneous systems [26].

\section{Conclusions}

In summary we can conclude that interpenetrating polymer networks of different morphological structures can be obtained using tri- tetra- and pentaacrylate monomers for photopolymerization in poly(lactic acid) matrix. The polyacrylate/PLA IPN's are heterogeneous - the inclusions and aggregates of different size have been observed under optical microscope.

Numerous factors influence the rate of photopolymerization besides of mixture composition: functionality of monomer, components miscibility and phase separation, intermolecular interactions, crystallinity, macrochain flexibility and mobility. Moreover, it was found that the initial sample morphology has strong effect on polymerization and photocuring kinetics influences the final network structure and properties.

On the basis of FTIR kinetics studies, it was found that PETeA exhibited the highest rate of reaction in nonplasticized PLA, whereas PETA photopolymerized fastest in PLA in the presence of PEG. It can be concluded that the number of functional groups and 
chemical structure of monomers plays an important role in the course of photopolymerization of acrylates in PLA matrix. Moreover, the decrease of PLA crystallinity in ternary composition facilitates the photopolymerization.

Gravimetric estimation of insoluble gel amount proves that a significant amount of PLA $(\sim 50-90 \%)$ participates in the photocrosslinking process. It means that full-IPN has been obtained resulting of competitive processes. The following mechanisms of interpenetrating network formation have to be taken into account: physical entanglement and chemical reaction because PLA becomes insoluble (only few $\%$ sol fractions were obtained from most samples). Chemical reactions comprise the grafting, simple recombination of macroradicals from both polymeric components as well as transesterification between PLA and monomer containing hydroxyl groups (PETA, DPEPA).

The benefits of this photochemical process are high rates, relatively high conversion degrees, conditions acceptable for industry (room temperature, air atmosphere). In plasticized networks, PEG crystallites were created, what was the reason of insufficient plasticization effect.

FTIR spectroscopy appeared as a convenient, powerful tool for the investigation of photopolymerization kinetics of multifunctional acrylate monomers alone, in linear polymer matrix as well as in plasticized formulations.

The plasticization effect by addition of PEG has been rather weak. In the case of ternary blends, the various factors influence the final properties of networks depending on the polymerization kinetics (composition, plasticizer presence, phase separation, partial crystallization of components and high crosslinking degree).

Finally, it should be added, that owing to the high efficiency of crosslinking, these networks composed of polyacrylate/PLA (1:1) can be recommended as protective surface layers, which are simultaneously ecological materials. The studies of IPN with lower amount $(<50 \%)$ of acrylate in PLA matrix will be conducted in the future for finding the optimal content for good physical/mechanical/barrier properties and biodegradability.

\section{Acknowledgements}

Authors thank Barbara Góralczyk from Department of Pathophysiology, Collegium Medicum, Nicolaus Copernicus University, Bydgoszcz, Poland for making microscopic images.

\section{References}

[1] Foltynowicz Z., Jakubiak P.: Polylactid acid - Biodegradable polymer obtained from vegetable resources. Polimery, 47, 769-774 (2002).

[2] Duda A., Penczek S.: Polylactide [poly(lactic acid)]: Synthesis, properties and applications. Polimery, 48, 16-27 (2003).

[3] Nieddu E., Mazzucco L., Gentile P., Benko T., Balbo V., Mandrile R., Ciardelli G.: Preparation and biodegradation of clay composites of PLA. Reactive and Functional Polymers, 69, 371-379 (2009).

DOI: $10.1016 /$ j.reactfunctpolym.2009.03.002

[4] Gupta B., Revagade N., Hilborn J.: Poly(lactic acid) fiber: An overview. Progress in Polymer Science, 32, 455-482 (2007).

DOI: 10.1016/j.progpolymsci.2007.01.005

[5] Rasal R. M., Janorkar A. V., Hirt D. E.: Poly(lactic acid) modifications. Progress in Polymer Science, 35, 338-356 (2010). DOI: $10.1016 /$ j.progpolymsci.2009.12.003

[6] Krasowska K., Brzeska J., Rutkowska M., Dacko P., Sobota M., Kowalczuk M.: The effect of poly(D,Llactide) modification with poli[(R,S)-3-hydroxybutyrate] on the course of its degradation in natural environments. Polimery, 53, 730-736 (2008).

[7] Athanassiou A., Lassithiotaki M., Anglos D., Georgiu S., Fotakis C.: A comparative study of the photochemical modifications effected in the UV laser ablation of doped polymer substrates. Applied Surface Science, 154-155, 89-94 (2000).

DOI: $10.1016 / \mathrm{S} 0169-4332(99) 00380-3$

[8] Mao C., Zhao W. B., Zhu A. P., Shen J., Lin S. C.: A photochemical method for the surface modification of poly(vinyl chloride) with $O$-butyrylchitosan to improve blood compatibility. Process Biochemistry, 39, 11511157 (2004). DOI: 10.1016/S0032-9592(03)00225-5

[9] Bahners T., Häßler R., Gao S-L., Mäder E., Wego A., Schollmeyer E.: Photochemical surface modification of PP for abrasion resistance. Applied Surface Science, 255, 9139-9145 (2009). DOI: 10.1016/j.apsusc.2009.06.118

[10] Paczkowski J.: Polymer photochemistry. Theory and applications (in Polish). Nicolaus Copernicus University Publishing. Torun (2003).

[11] Kaczmarek H.: Photopolymerization of trimethylolpropane triacrylate in a matrix of linear-polymers. Polimery, 40, 333-340 (1995). 
[12] Fouassier J. P., Rabek J. F.: Radiation curing in polymer science and technology. Elsevier, London (1993).

[13] Narayanan V., Scranton A. B.: Photopolymerization of composites. Trends in Polymer Science, 5, 415-419 (1997).

[14] Decker C.: New developments in UV radiation curing of protective coatings. Surface Coatings International Part B: Coatings Transactions, 88, 9-17 (2005). DOI: $10.1007 / \mathrm{BF} 02699702$

[15] Andrzejewska E.: Photopolymerization kinetics of multifunctional monomers. Progress in Polymer Science, 26, 605-665 (2001). DOI: $10.1016 / \mathrm{S} 0079-6700(01) 00004-1$

[16] Kaczmarek H., Ołdak D., Szalla A.: Networks of photocrosslinked poly(meth)acrylates in linear poly(vinyl chloride). Journal of Applied Polymer Science, 86, 3725-3734 (2002).

DOI: 10.1002/app.11434

[17] Kaczmarek H., Decker C.: Interpenetrating polymer networks. I. Photopolymerization of multiacrylate systems. Journal of Applied Polymer Science, 54, $2147-$ 2156 (1994).

DOI: 10.1002/app.1994.070541317

[18] Piorkowska E., Kulinski Z., Galeski A., Masirek R.: Plasticization of semicrystalline poly(L-lactide) with poly(propylene glycol). Polymer, 47, 7178-7188 (2006). DOI: $10.1016 /$ j.polymer.2006.03.115

[19] Martin O., Avérous L.: Poly(lactic acid): Plasticization and properties of biodegradable multiphase systems. Polymer, 42, 6209-6219 (2001). DOI: $10.1016 / \mathrm{S} 0032-3861(01) 00086-6$
[20] Feldstein M. M.: Peculiarities of glass transition temperature relation to the composition of $\operatorname{poly}(N$-vinyl pyrrolidone) blends with short chain poly(ethylene glycol). Polymer, 42, 7719-7726 (2001).

DOI: $10.1016 / \mathrm{S} 0032-3861(01) 00225-7$

[21] Li H., Huneault M. A.: Effect of nucleation and plasticization on the crystallization of poly(lactic acid). Polymer, 48, 6855-6866 (2007).

DOI: $10.1016 /$ j.polymer.2007.09.020

[22] Agrawal A., Saran A. D., Rath S. S., Khanna A.: Constrained nonlinear optimization for solubility parameters of poly(lactic acid) and poly(glycolic acid)-validation and comparison. Polymer, 45, 8603-8612 (2004).

DOI: $10.1016 /$ j.polymer.2004.10.022

[23] Schenderlein S., Lück M., Müller B. W.: Partial solubility parameters of poly(D,L-lactide-co-glycolide). International Journal of Pharmaceutics, 286, 19-26 (2004).

DOI: $10.1016 /$ j.ijpharm.2004.07.034

[24] Lindvig T., Michelsen M. L., Kontogeorgis G. M.: A Flory-Huggins model based on the Hansen solubility parameters. Fluid Phase Equilibria, 203, 247-260 (2002).

DOI: 10.1016/S0378-3812(02)00184-X

[25] Mamun C. K.: Microstructures in interpenetrating polymer networks. Superlattices and Microstructures, 23, 1107-1115 (1998). DOI: $10.1006 /$ spmi.1998.0566

[26] Athawale V. D., Pillay P. S.: Interpenetrating polymer networks based on uralkyd-butylmethacrylate. Reactive and Functional Polymers, 50, 1-8 (2001). DOI: $10.1016 / \mathrm{S} 1381-5148(01) 00084-0$ 